\title{
Developing a clinical decision support system for paediatric shoulder instability
}

\author{
Amina Yaqub \\ Keele University \\ School of Allied Health \\ Professions/University \\ Hospitals of Leicester \\ amina.yaqub@hotmail.co.uk
}

\author{
Michael McCluskey \\ University of Liverpool \\ School of Health Sciences \\ m.mccluskey@liverpool.ac.uk
}

\author{
Edward Chadwick \\ University of Aberdeen \\ School of Engineering \\ edward.chadwick@abdn.ac.uk
}

\author{
Fraser Philp \\ University of Liverpool/ \\ School of Health \\ Sciences \\ f.philp@liverpool.ac.uk
}

\begin{abstract}
Paediatric shoulder instability is a complex condition which may benefit from clinical decision support systems. The study aims were to elicit the types of information used in clinical decision making, and get early stakeholder involvement to inform the design of a decision support system. Knowledge elicitation was achieved using an online questionnaire distributed to registered physiotherapists. A wide range of factors used in clinical decision-making process were identified. These may inform the development of a clinical decision support system in conjunction with clinicians self-reported knowledge regarding 3D motion analysis and its perceived. This early stakeholder involvement is important for ensuring that subsequently developed systems are clinically applicable.
\end{abstract}

User centre design; knowledge elicitation; stakeholder involvement; clinical decision support systems; paediatric shoulder instability; rehabilitation; physiotherapy; clinical decision making; movement analysis.

\section{INTRODUCTION}

Shoulder instability (SI) that is, complete or partial dislocation of the shoulder, is common in children (overall incidence of 23.9 to $164.4 / 100,000$-person years in 10 to 16-year olds, (Leroux et al., 2015)). Current methods for diagnosing and treating $\mathrm{SI}$ in children are inadequate (Philp et al., 2021). Time to diagnosis can be delayed by up to two years and failure rates with rehabilitation are high with $70 \%$ to $90 \%$ of children continuing to suffer recurrent instability within 1 to 2 years after their initial instability episode (Longo et al., 2016). SI can result in pain, decreased movement and disability. Recurrent SI can damage the shoulder joint resulting in early arthritis onset (odds ratio 19.3) (Deitch et al., 2003, Marx et al., 2002).

\subsection{CONCEPT OF A TOOL FOR CLINICAL DECISION MAKING IN PAEDIATRIC SHOULDER INSTABILITY}

Mechanisms for $\mathrm{SI}$ are not well understood and clinical decision making is complex. Clinical Decision Support Systems (CDSS) may help in reducing time to diagnosis and improve patient outcomes. CDSS involving, three-dimensional (3D) motion analysis (figure 1) and clinical algorithms for imaging selection have been shown to improve diagnostic accuracy in $\mathrm{SI}$ and other upper-limb conditions (Moroder et al., 2020, Brunner et al.,
2020). Despite their utility, they are not widely used in practice. A possible reason for this is the lack of early end-user involvement and explicit mapping of information used in clinical decision-making.

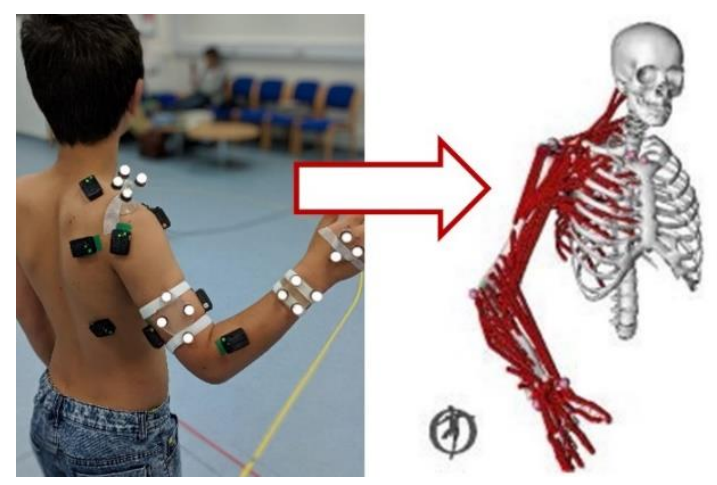

Figure 1: 3D motion analysis of a child, data from which is then inputted into a musculoskeletal OpenSim model

Early stakeholder involvement is imperative for ensuring a CDSS is clinically applicable (Lane et al., 2016). This is particularly important for complex conditions, such as $\mathrm{SI}$, where there are potentially significant amounts of information that may be derived from multiple sources e.g. CDSS involving 3D-movement analysis. It is also unclear what information would be useful in clinical decision making. There is a risk that the user will be overwhelmed with information and interfaces which are complex to navigate or present information that is redundant or difficult to interpret, will limit 
engagement (Jaspers et al., 2011). Performance of a CDSS is also dependent on the availability of appropriate patient characteristics and the systems knowledge base. In paediatric shoulder instability, it is not clear which patient characteristics should be included and if there is a sufficient knowledge base for the system to refer to.

The aims of this study are therefore to 1) elicit the types of information used for clinical decision making in paediatric shoulder instability and 2) get early stakeholder involvement prior to development, to inform the design of a decision support system, which may integrate 3D movement analysis data.

\section{METHODS}

Ethical approval was gained from the School of Allied Health Professions Student Project Ethics Committee, Keele University. Knowledge elicitation was carried out using a web-based questionnaire (at https://doi.org/10.5281/zenodo.5018670). The questionnaire was developed by the clinical and non-clinical authors, informed by the literature and evaluated for face validity prior to recruitment. Participants were recruited over a two-month period, from November 2019 to January 2020, through social media platforms and special interest/ professional group mailing lists. Participants were included if they were qualified physiotherapists, currently working adult and paediatric SI patients. Participants were excluded if they were not registered physiotherapists or did not assess patients with SI.

\section{RESULTS}

A total of 19 responses (12F:7M) were received. Respondents had been qualified for an average of 14.6 years (SD 8.1) and worked primarily in orthopaedic settings. Eighteen respondents worked in the UK and one worked in Egypt. Only one participant had previous experience working in a movement analysis service.

\subsection{Results for diagnostic information}

Six participants did not report using any classification systems. The Stanmore classification/Bayley triangle (Lewis et al., 2004) framework the most frequently $(n=11)$, followed by the Gerber and Nyffeler (Gerber and Nyffeler, 2002) $(n=2)$, and the Thomas and Matsen (Thomas, 1989) frameworks $(n=1)$. A summary of the subjective factors' clinicians found important for diagnosing paediatric shoulder instability, and frequency of selection, are reported in Table 1. The number of factors selected by any individual ranged from five to 16 , with a mode of eight. No single subjective or objective factor was identified universally by all respondents. A summary of the objective factor's clinicians found important for diagnosing $\mathrm{SI}$, and frequency of selection, are reported in Table 2. The number of factors selected as being important for shoulder instability by any individual ranged from two to 16 , with a mode of seven.

Table 1: Frequency of subjective factor selection

\begin{tabular}{|l|c|}
\hline Subjective factor & $\begin{array}{c}\text { Number of } \\
\text { responses }\end{array}$ \\
\hline Previous episode(s) of SI & 18 \\
\hline Sensation(s) of SI & 17 \\
\hline $\begin{array}{l}\text { Previous shoulder injury, Injury } \\
\text { mechanism consistent with SI }\end{array}$ & 16 \\
\hline $\begin{array}{l}\text { Age, Previous surgery related to } \\
\text { instability }\end{array}$ & 15 \\
\hline Loss of Function & 13 \\
\hline Pain, Weakness & 11 \\
\hline Previous investigations & 10 \\
\hline Gender & 9 \\
\hline Previous treatment & 8 \\
\hline History of repetitive micro trauma, & 7 \\
\hline Family history of SI & 3 \\
\hline Locking, Deformity & 2 \\
\hline Stifness & 1 \\
\hline Catching, Swelling, Clicking & \\
\hline
\end{tabular}

Factors separated by (,)

Table 2: Frequency of objective factor selection

\begin{tabular}{|l|c|}
\hline \multicolumn{1}{|c|}{ Objective factor } & $\begin{array}{c}\text { Number of } \\
\text { responses }\end{array}$ \\
\hline Apprehension relocation test & 16 \\
\hline Sulcus Sign & 14 \\
\hline AROM Apprehension & 12 \\
\hline $\begin{array}{l}\text { Beightons score greater than or equal } \\
\text { to 4/9 }\end{array}$ & 11 \\
\hline $\begin{array}{l}\text { Loss of rotation (internal \& external), } \\
\text { Posture e.g. asymmetry, scapular } \\
\text { winging, atrophy, passive range of } \\
\text { movement apprehension }\end{array}$ & 10 \\
\hline $\begin{array}{l}\text { Loss of active abduction, Posterior } \\
\text { apprehension, Scapula Dyskinesis }\end{array}$ & 9 \\
\hline Inferior apprehension & 8 \\
\hline $\begin{array}{l}\text { Anterior shift load, Loss of active } \\
\text { flexion }\end{array}$ & 6 \\
\hline $\begin{array}{l}\text { Drawer tests, Loss of active } \\
\text { adduction, Loss of active extension }\end{array}$ & 5 \\
\hline $\begin{array}{l}\text { Pain/stiffness with passive range of } \\
\text { movement, posterior shift load }\end{array}$ & 4 \\
\hline Tenderness on palpation & 3 \\
\hline Release Test & 2 \\
\hline $\begin{array}{l}\text { Arc Test, Hyperabduction test, Jerk } \\
\text { test, Shoulder Symptom Modification } \\
\text { Procedure, Rotator cuff weakness }\end{array}$ & 1 \\
\hline
\end{tabular}

\subsection{Results for rehabilitation protocols used}

Overall seven protocols for rehabilitation were identified. Only two respondents reported not using any protocols. The Derby Shoulder Instability Rehabilitation Programme was the most frequently identified (Bateman et al., 2019) $(n=9)$, followed by 
Consultant developed/led protocols $(n=7)$, Department specific protocols $(n=3)$, the Watson Multidirectional Instability Program (Watson et al., 2016, Watson et al., 2017) ( $n=2)$, Trust specific protocols $(n=1)$, Individualised protocols $(n=1)$ and protocols based on external courses $(n=1)$.

\subsection{Results for respondent's knowledge and understanding, and perceived usefulness of movement analysis}

Overall results are presented in figure 5 . Respondents reported having average $(47.4 \%)$ or good knowledge (42.1\%) of biomechanical and musculoskeletal models and having poor or very poor knowledge or ability (more than 60\%) regarding 3D movement analysis and interpretation of electromyography (EMG) and kinetic data. The most frequently selected answer was that respondents neither agreed nor disagreed that kinetic data, EMG and 3D movement data would improve their current understanding, diagnosis and management of paediatric SI. The use of biomechanical and musculoskeletal models was viewed positively by the majority of respondents with $42.1 \%$ and $15.8 \%$ of respondents agreeing or strongly agreeing respectively.

Figure 5. Respondents knowledge and understanding of movement analysis and perceived usefulness of motion analysis in improving understanding, assessment and management of paediatric shoulder instability

\section{A. Respondents knowledge and understanding of movement analysis}

How would you rate your knowledge and understanding of BIOMECHANICAL and MUSCULOSKELETAL MODELS?

How would you rate your ability to interpret and analyse KINETIC DATA

How would you rate your ability to interpret and analyse ELECTROMYOGRAPHY (EMG) DATA?

How would you rate your knowledge and understanding of 3D MOVEMENT ANALYSIS?

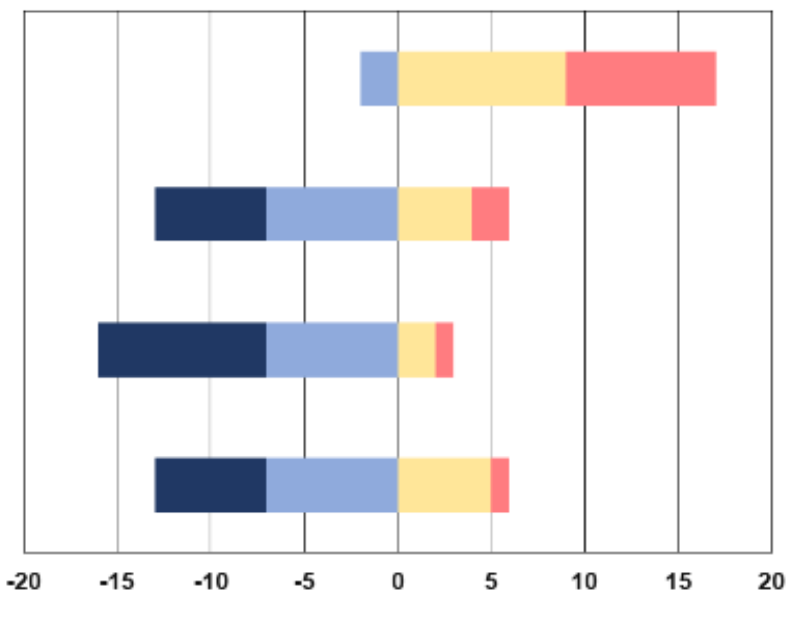

- Very Poor = Poor $=$ Average $\mid$ Good $\mid$ Excellent

\section{B. Respondents perceived usefulness of motion analysis in improving understanding, assessment and} management

BIO MECHANICAL and MUSCULOSKELETAL MODELS would help in improving our current understanding. diagnosis and management of paediatric shoulder instability

KINETIC DATA e.g. forces would help in improving our current understanding, diagnosis and management of paediatric shoulder instability

ELECTROMYOGRAPHY (EMG) DATA would help in improving our current understanding, diagnosis and management of paediatric shoulder instability

3D MOVEMENT ANALYSIS would help in improving our current understanding, diagnosis and management of paediatric shoulder instability

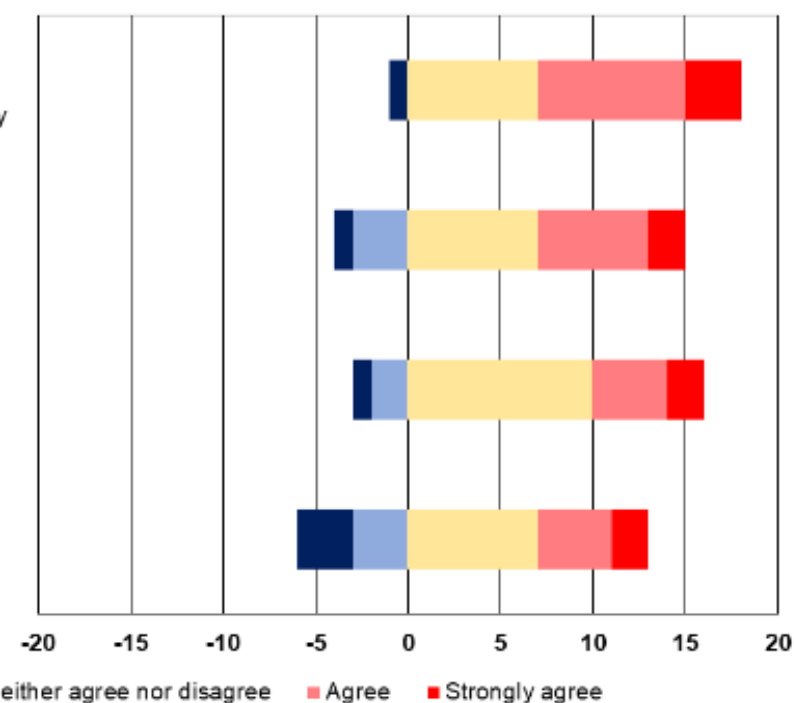

-Strongly disagree $=$ Disagree Neither agree nor disagree $\|$ Agree $\|$ Strongly agree

\section{DISCUSSION}

The aims of this study were to 1) elicit the types of information used for clinical decision making in paediatric shoulder instability and 2) get stakeholder involvement in the early stages of development, to inform the design of a decision support system, which may integrate 3D movement analysis data. Our results indicate that there is good rationale for the use of a CDSS which may ultimately reduce practice variation and improve patient outcomes. We have also identified explicit factors that could be 
used for informing the initial development of a CDSS, associated interface and future work.

There was considerable variation in the number of selected subjective and objective factors. This is consistent with other published studies reporting between one to 16 factors (Kuhn et al., 2011). The most frequently selected subjective and objective factors were history or sensation of $\mathrm{SI}$, and positions which made the patient report SI. It is not clear what the minimum number of criteria needed to accurately diagnose shoulder instability is, or which factors are considered the most important by individual therapists. This poses challenges for the development of a CDSS, as a large number of factors will increase imputation burden and limit clinical integration. Additionally, for cases in which clinicians use fewer factors, the accuracy of the CDSS will be negated if required factors not deemed important by individual clinicians are omitted. Further work is required to identify the agreed minimum number of factors required for accurate diagnosis. This may be achieved by using alternate methodologies such as Delphi and nominal focus group techniques.

Agreed classification systems, management pathways and suitable knowledge base are required for an effective CDSS. Existing systems are inadequate given their complexity and poor evidence base (Warby et al., 2018). Furthermore, they are prone to error as 1) there is poor agreement between patient reported and clinician derived factors (Kuhn et al., 2011) 2) "objective factors" e.g. joint integrity tests, lack sensitivity and specificity and are not always equivalent true physiological or biomechanical measures. This may explain why these systems have not been universally adopted e.g. no classification framework was the $2^{\text {nd }}$ most frequent response $(n=6)$.

It was identified that whilst motion analysis may enhance our understanding of paediatric shoulder instability, a lack of experience and knowledge regarding motion analysis may have affected clinicians perceived usefulness of these methods. Implementation of 3D motion analysis into routine clinical pathways has been done successfully in other domains (Laracca et al., 2014). Currently, however movement analysis requires referral into a specialist centres and so their integration into the clinical pathway requires further investigation. Respondents neither agreed nor disagreed about the use of 3D movement, kinetic and EMG data for the same purpose. These results are surprising given the interpretation of biomechanical/musculoskeletal models are dependent on 3D movement, kinetic and EMG data. A possible reason for this may be limited experience and knowledge of these measurement methods. If a CDSS is to incorporate the information derived from 3D motions analysis, future work or training may be needed to familiarise clinicians with these measurement methods and identify what additional information derived from motion analysis would be useful.

\subsection{Limitations}

We were unable to report a response rate as the questionnaire was distributed using social media platforms and special interest/ professional group mailing lists. Whilst not all physiotherapists manage patients with shoulder instability, it is recognised that our proportionate sample size is small given there are more than 50,000 registered physiotherapists in the UK (HCPC, 2017). Patients with SI may also be managed by other healthcare professionals and it would be beneficial to include all relevant stakeholders in the CDSS design process. Whilst a larger sample size may have highlighted additional factors, it is unlikely that these will be helpful given the considerable variation in practice already observed. Selfreported knowledge of clinicians against their actual performance was not verified and may account for our results. Despite a correlation between perceived and actual knowledge (Chan and Zang, 2007), healthcare professionals overestimate their actual knowledge and ability when less experienced and operating in new domains (Caspi et al., 2006). Further work is therefore required to establish the actual knowledge and ability of clinicians to interpret information derived from motion analysis, if this is to be integrated in a CDSS.

\section{CONCLUSION}

There are considerable variations in practice, for diagnosis and management of assessment, and this may be addressed by the development of an appropriate system. Stakeholder involvement identified that overall respondents agreed that biomechanical and musculoskeletal models would help to improve assessment and management of paediatric shoulder instability. It is recognised that prior to development of a clinically applicable systems, further work is needed to develop consensus and minimally required criteria for the assessment and diagnosis of paediatric shoulder instability. Furthermore, robust classification and management paradigms are required as these will serve as the effectiveness of a CDSS is dependent on quality of the knowledge base. 


\section{REFERENCES}

BATEMAN, M., OSBORNE, S. E. \& SMITH, B. E. 2019. Physiotherapy treatment for atraumatic recurrent shoulder instability: Updated results of the Derby Shoulder Instability Rehabilitation Programme. Journal of arthroscopy and joint surgery, 6, 35-41.

BRUNNER, M. C., SHEEHAN, S. E., YANKE, E. M., SITTIG, D. F., SAFDAR, N., HILL, B., LEE, K. S., ORWIN, J. F., VANNESS, D. J. \& HILDEBRAND, C. J. 2020. Joint Design with Providers of Clinical Decision Support for Value-Based Advanced Shoulder Imaging. Applied clinical informatics, 11, 142-152.

CASPI, O., MCKNIGHT, P., KRUSE, L., CUNNINGHAM, V., FIGUEREDO, A. J. \& SECHREST, L. 2006. Evidence-based medicine: discrepancy between perceived competence and actual performance among graduating medical students. Medical Teacher, 28, 318-325.

CHAN, M. F. \& ZANG, Y. L. 2007. Nurses' perceived and actual level of diabetes mellitus knowledge: results of a cluster analysis. Journal of Clinical Nursing, 16, 234-242.

DEITCH, J., MEHLMAN, C. T., FOAD, S. L., OBBEHAT, A. \& MALLORY, M. 2003. Traumatic anterior shoulder dislocation in adolescents. The American journal of sports medicine, 31, 758-763.

GERBER, C. \& NYFFELER, R. W. 2002. Classification of glenohumeral joint instability. Clin Orthop Relat Res, 65-76.

HCPC. 2017. Total number of registered Physiotherapists and the number of supplementary and independent prescribers - November 2017 [Online]. Available: $\quad$ http://www.hcpcuk.co.uk/resources/freedom-of-informationrequests/2017/total-number-of-registeredphysiotherapists-and-the-number-ofsupplementary-and-independentprescribers---november-2017/ [Accessed 25th February 2020].

JASPERS, M. W., SMEULERS, M., VERMEULEN, H. \& PEUTE, L. W. 2011. Effects of clinical decision-support systems on practitioner performance and patient outcomes: a synthesis of high-quality systematic review findings. J Am Med Inform Assoc, 18, 32734.

KUHN, J. E., HELMER, T. T., DUNN, W. R. \& THROCKMORTON, V. T. 2011. Development and reliability testing of the frequency, etiology, direction, and severity (FEDS) system for classifying glenohumeral instability. J Shoulder Elbow Surg, 20, 548-56.

LANE, S., O'RAGHALLAIGH, P. \& SAMMON, D. 2016. Requirements gathering: the journey. Journal of Decision Systems, 25, 302-312.

LARACCA, E., STEWART, C., POSTANS, N. \& ROBERTS, A. 2014. The effects of surgical lengthening of hamstring muscles in children with cerebral palsy-the consequences of pre-operative muscle length measurement. Gait \& posture, 39, 847-851.

LEROUX, T., OGILVIE-HARRIS, D., VEILLETTE, C., CHAHAL, J., DWYER, T., KHOSHBIN, A., HENRY, P., MAHOMED, N. \& WASSERSTEIN, D. 2015. The epidemiology of primary anterior shoulder dislocations in patients aged 10 to 16 years. Am J Sports Med, 43, 2111-7.

LEWIS, A., KITAMURA, T. \& BAYLEY, J. 2004. (ii) The classification of shoulder instability: new light through old windows! Current Orthopaedics, 18, 97-108.

LONGO, U. G., VAN DER LINDE, J. A., LOPPINI, M., COCO, V., POOLMAN, R. W. \& DENARO, V. 2016. Surgical Versus Nonoperative Treatment in Patients Up to 18 Years Old With Traumatic Shoulder Instability: A Systematic Review and Quantitative Synthesis of the Literature. Arthroscopy, 32, 944-52.

MARX, R. G., MCCARTY, E. C., MONTEMURNO, T. D., ALTCHEK, D. W., CRAIG, E. V. \& WARREN, R. F. 2002. Development of arthrosis following dislocation of the shoulder: A case-control study. Journal of Shoulder and Elbow Surgery, 11, 1-5.

MORODER, P., DANZINGER, V., MAZIAK, N., PLACHEL, F., PAULY, S., SCHEIBEL, M. \& MINKUS, M. 2020. Characteristics of functional shoulder instability. Journal of Shoulder and Elbow Surgery, 29, 68-78.

PHILP, F., FAUX-NIGHTINGALE, A., WOOLLEY, S., DE QUINCEY, E. \& PANDYAN, A. 2021. Implications for the design of a Diagnostic Decision Support System (DDSS) to reduce time and cost to diagnosis in paediatric shoulder instability. BMC Medical Informatics and Decision Making, 21, 78.

THOMAS, S. C. 1989. An approach to the repair of avulsion of the glenohumeral ligaments in the management of traumatic anterior glenohumeral instability. The Journal of bone and joint surgery. American volume, 71, 506-513.

WARBY, S. A., FORD, J. J., HAHNE, A. J., WATSON, L., BALSTER, S., LENSSEN, R. \& PIZZARI, T. 2018. Comparison of 2 Exercise Rehabilitation Programs for Multidirectional Instability of the 
Glenohumeral Joint: A Randomized Controlled Trial. The American journal of sports medicine, 46, 87-97.

WATSON, L., WARBY, S., BALSTER, S., LENSSEN, R. \& PIZZARI, T. 2016. The treatment of multidirectional instability of the shoulder with a rehabilitation program: part 1. Shoulder \& elbow, 8, 271-278.
WATSON, L., WARBY, S., BALSTER, S., LENSSEN, R. \& PIZZARI, T. 2017. The treatment of multidirectional instability of the shoulder with a rehabilitation programme: Part 2. Shoulder \& elbow, 9, 46-53. 